\title{
LA FILOSOFÍA DE LA CIENCIA, EL REALISMO CIENTÍFICO Y LA PERVIVENCIA DEL EMPIRISMO LÓGICO
}

\author{
PHILOSOPHY OF SCIENCE, SCIENTIFIC REALISM \\ AND THE SURVIVAL OF LOGICAL EMPIRICISM
}

\author{
Juan Vicente Mayoral $^{1}$ \\ DOI: 10.26754/ojs_arif/a.rif.201924087
}

Mauricio Suárez, Filosofía de la ciencia: historia y práctica. Madrid: Tecnos, 2019. 216 pp. $15 €$.

Los manuales de filosofía de la ciencia suelen intentar proporcionar al estudiante una instantánea del estado de la disciplina en el momento en que son escritos. Su eficacia como recurso para la educación de varias generaciones depende de varios factores, como la precisión alcanzada en la descripción, el momento atravesado por la disciplina o el número de actualizaciones a los que se los somete. Sea como fuere, suelen ser instrumentos útiles para el docente y el alumnado y algunos logran resistir el paso del tiempo sin resultar obsoletos.

Al mismo tiempo, sin embargo, algunos de ellos suelen ser algo parecido a extensos informes protocolares algo desprovistos de vida, en los que el autor o autores permanecen voluntariamente, como observadores interesados en la materia, al margen de la descripción. Su participación visible se limita a la del autor o autores con habilidad para comprender la disciplina en cuestión, para comunicarla y, ocasionalmente, para dotar de mayor peso a ciertos temas que son de su interés, en detrimento de otros. Son menos frecuentes los manuales de filosofía de la ciencia que intentan hacer ver la disciplina bajo un punto de vista participante en el que la intención y las tendencias y opiniones sobre los autores se hacen explícitas, a la vez que se repasan las doctrinas de los filósofos de la

\footnotetext{
1 Estoy en deuda con David Teira, Mariano Sanjuán y Carlos Solís por algunos comentarios de gran ayuda sobre una versión anterior del texto.
} 
ciencia mejor conocidos y los problemas que ha suscitado la materia. Este punto de vista, cuando es solo visible a través del diseño del manual, no queda patente para el lector ocasional ni para aquel cuya formación depende en parte de él. No me atrevería a asegurar, sin embargo, que esos dos tipos de lectores prefieran una introducción aséptica a la disciplina a un comentario (no desprovisto de rigor y detalle) sobre las idas y venidas de una materia cada vez más compleja y diversificada. Despertar el interés sobre ella no consiste solamente en mostrar su utilidad, sino también en desvelar las opiniones de quienes ya están interesados en ella, que priman unos aspectos sobre otros y que tienen los conocimientos necesarios para ofrecer una versión informada.

El manual de Mauricio Suárez, Filosofía de la ciencia: historia y práctica, intenta superar el enfoque más aséptico y ofrecer una visión más participante. Para ello, muestra su preferencia por dos tesis principales a la vez que describe contenidos básicos de reconocido interés para la formación de un alumnado interesado en la materia (o un lector ocasional). La primera tesis tiene un carácter histórico: la filosofía de la ciencia del siglo pasado debe una buena parte de sus recursos, de su vocabulario y de las soluciones iniciales a los problemas filosóficos del estudio del método científico a una corriente específica, el positivismo lógico del Círculo de Viena, la Sociedad Berlinesa de Filosofía Científica y sus desarrollos posteriores, con esos filósofos en el exilio (p. 13). ${ }^{2}$ Esta es una corriente que habitualmente se nombra mediante una cierta variedad de denominaciones que no son completamente equivalentes: "positivismo lógico", "neopositivismo", "empirismo lógico" o "concepción heredada". Suárez, que opta por una denominación basada en la utilidad antes que en la precisión histórica (véanse las pp. 19-22), describe la corriente en el primer capítulo y en la introducción a la primera parte ("El programa clásico neopositivista") y engloba en ello una visión renovada, alejada de perspectivas históricas previas sobre ella que la contemplaban como una isla de terreno filosóficamente estéril obsesionada con la eliminación de toda consideración metafísica. ${ }^{4}$ La relación del trabajo de sus participantes con el de otros previos y ajenos a la misma pone de manifiesto algo que, desde la obra de Michael Friedman, o de George Reisch, junto con otros autores, se ha

\footnotetext{
2 A menos que se indique lo contrario, todas las referencias a páginas en el texto son al libro de Suárez.

3 Para una exposición reciente del origen de dichas denominaciones y de sus diferencias, véase Creath 2017, sec. 1; véase también Earman 1993, p. 33, n. 1.

4 Sobre esa actitud hacia la corriente, véase Friedman 1999, pp. xii-xiii.
} 
defendido con cada vez mayor fuerza: que la filosofía de la ciencia del positivismo lógico merece una mayor atención que la que habitualmente se le ha prestado. Este manual es una buena prueba de que esta opción historiográfica es cada vez más atractiva.

La relevancia histórica del positivismo lógico se muestra en este manual, por ejemplo, cuando el lector llega al final de la primera parte (p. 101) y, tras repasar los comienzos y los contenidos principales de dos de los focos mayoritarios de interés en filosofía de la ciencia, la teoría de la explicación (caps. 2-3) y la de la confirmación científica (caps. 4-5), Suárez subraya una vez más que todo cuanto aquel ha averiguado acerca de ambas se debe, en buena medida, a la labor de los filósofos lógico-positivistas. La conclusión tiene un cierto sabor a paradoja, porque, sin el trabajo de otros autores posteriores al positivismo lógico o la concepción heredada, esos mismos compromisos filosóficos que ponen en marcha el estudio de los problemas de la confirmación y de la explicación científica tornan el programa en un proyecto ineficaz. Con todo, queda claro que la filosofía de la ciencia no puede desdeñar dicho programa, porque ni el interés en problemas como los mencionados ni muchas de las herramientas empleadas (de la lógica a la teoría de la probabilidad) estarían sobre la mesa aún hoy de no ser por la esforzada labor de los filósofos del Círculo de Viena o de la Sociedad Berlinesa. Es más, incluso algunas reacciones críticas les deben su medio de expresión. Al abogar por el legado lógico-positivista, Suárez muestra que incluso la perspectiva sobre la inconmensurabilidad de uno de los azotes del empirismo, el también vienés Paul K. Feyerabend, se expresa en el vocabulario del empirismo lógico y es más clara que la de Thomas Kuhn por esa misma razón (pp. 126-127). ${ }^{5}$

La segunda tesis es filosófica y está constituida por la perspectiva realista en filosofía de la ciencia y, más en particular, la del realismo experimental, o realismo de entidades, como habitualmente se la conoce, arraigada en el pensamiento de Ian Hacking y de Nancy Cartwright (con quien el autor del manual ha colaborado a menudo) ${ }^{6}$ y que Suárez ha contribuido a desarrollar (véanse las pp. 108 y 187). Esta preferencia es explicada en más de una ocasión a lo largo del libro y principalmente

\footnotetext{
5 “En este tema, en concreto", dice Suárez (p. 126), "la deuda de Feyerabend con el Círculo de Viena es palpable, y la única fórmula que, según este autor, permite una definición clara y sin ambigüedades de la inconmensurabilidad de dos teorías científicas recurre, en última instancia, a las categorías de la llamada concepción heredada de las teorías científicas que, en esencia, desarrollasen los neopositivistas".

${ }^{6}$ Sobre esa colaboración, véase, e.g., Peschard y Van Fraassen 2018, pp. 28-32.
} 
en aquellos pasajes en que Suárez expone puntos de inflexión en el desarrollo de la disciplina. Como antes decíamos, el final de la primera parte (p. 101) es uno de ellos y en él nuestro autor muestra que el programa del positivismo lógico se da por agotado en la medida que las ideas de confirmación y de explicación requieren atender a algo más que a la estructura puramente lógica de una relación entre enunciados. Los compromisos con una ontología de base en las teorías científicas y las afirmaciones de nuestra capacidad de acceso epistémico a ella son motivos de discusión y forman parte del material expuesto críticamente en la segunda parte del libro, lo que desemboca en una exposición y defensa detallada del citado realismo experimental.

Navegamos, pues, en este manual, por buena parte de la filosofía de la ciencia del siglo pasado y los comienzos del actual gracias a una bien llevada combinación de exposición y tratamiento participante por parte del autor. Una ventaja de esta perspectiva es que ofrece un relato históricamente continuo a la par que muestra contenidos que deben ser explorados de manera sistemática si nuestros objetivos son pedagógicos. Un buen ejemplo de ello se deja ver en uno de los temas fundamentales del positivismo lógico: la teoría de la confirmación. Suárez se ocupa de ella en los capítulos 4 y 5 , una vez que ha explorado, en los dos capítulos previos (2-3) los entresijos y problemas de los modelos de Carl Hempel sobre explicación científica; en particular, de los dos conocidos modelos DN e IS (esto es, "deductivo-nomológico" e "inductivo-estadístico"; véanse las pp. 45-52).

La teoría de la confirmación nace al hilo de problemas (paradojas, en concreto) que trata Hempel, dada la definición de confirmación que ya ofreciera Jean Nicod, según la cual los casos positivos particulares de una cierta generalización empírica confirman esta última (véase Hempel 1945/1965). Si yo afirmo que todos los osos polares son blancos, cada nuevo oso polar blanco que me encuentre es una instancia confirmatoria de mi generalización inicial. Hempel pone de manifiesto algo aparentemente inocente sobre esta definición (que resulta razonable), aunque con ciertas consecuencias paradójicas. La confirmación según Nicod debería aplicarse no solo a mi generalización sobre los osos polares, sino a aquellas expresiones lógicamente equivalentes de ella; por ejemplo, a aquella que obedece a la regla lógica de contraposición y que dice que, si algo no es de color blanco, entonces no es un oso polar. Naturalmente, en este segundo caso, y obedeciendo al criterio de Nicod, cada nuevo objeto de un color diferente al blanco con el que me tope y que no sea un oso polar es una instancia confirmatoria de esta segunda afirmación general. El planeta Marte es, de este modo, una buena instancia confirmatoria, en tanto que ni es blanco ni es un oso polar. La cuestión es que, 
dada la supuesta y obvia capacidad de las instancias confirmatorias de avalar no solo las generalizaciones empíricas de las que son casos particulares sino también sus equivalencias lógicas, parece que el planeta Marte es capaz de confirmar la hipótesis de que todos los osos polares son blancos, lo que huele a resultado paradójico. Si sustituimos los osos polares y el color blanco por los cuervos y su acostumbrado color negro, tenemos el formato habitual de presentación de este problema por parte de Hempel y que Suárez asimismo expone en esos términos (véanse las pp. 76-81). ${ }^{7}$

No me adentraré en la solución de las paradojas, pues eso es algo que Suárez hace con brevedad y, al mismo tiempo, precisión y claridad pedagógicas, sino que me centraré en la manera en que el autor de este manual revela puntos de continuidad y discontinuidad dentro de la evolución de la teoría de la confirmación. Suárez muestra que un modo eficaz de abordar el problema dentro del mismo espíritu y programa positivista que da lugar al diagnóstico nace con la comprensión probabilista, bayesiana, de la confirmación, en la que, pese a hablar aún de la confirmación como una relación lógica entre enunciados, no perdemos de vista algo que hasta ahora no habíamos tenido demasiado en cuenta: el grado en que las instancias positivas de una hipótesis constituyen evidencia a favor de esta última. Esto tiene la ventaja de no derribar el argumento de base lógica de la teoría de la confirmación de Nicod y Hempel, pues respeta la idea de confirmación clásica y la referente a la equivalencia lógica (que para Hempel son dos condiciones imprescindibles), a la par que se enfrenta a la paradoja en sí (véanse las pp. 84, 89 y 93-94). De acuerdo con la perspectiva bayesiana (y, cabe subrayar, afín al positivismo lógico), la conclusión obtenida es válida: las instancias positivas confirman una hipótesis y sus equivalencias lógicas, pero allá donde exista un aire de paradoja — que no es tal, como ya reconocía el propio Hempel (1945/1965, pp. 18-20) - lo que ocurre simplemente es que la instancia confirma la generalización en un grado mínimo (pero lo hace, no obstante), mientras que lo hace en mayor grado en aquellos casos en los que no hay impresión de paradoja (para esta explicación, véanse las pp. 93-95). El aparato teórico del enfoque probabilista respalda esta interpretación.

Los capítulos de la teoría de la confirmación están así vinculados por un mismo conjunto de problemas. El primero ofrece una versión de las bases de la teoría de la confirmación en Hempel y muestra sus problemas (o resultados paradójicos, de nuevo) asociados, mientras que el segundo da un paso hacia los trabajos de

\footnotetext{
7 Este párrafo se basa en Hempel 1945/1965, pp. 10 y sigs.
} 
Rudolf Carnap y John Maynard Keynes sobre la confirmación cuantitativa de base probabilista y expone resultados que, en última instancia, conducen más allá de las concepciones estrictamente lógico-positivistas (véanse las pp. 85-93). Esto último aparece en la última sección del capítulo 5, titulada "Bayesianismo sin positivismo", y en la que se da solución, ya desde alternativas al espíritu lógico-positivista, a otra de las paradojas presentadas en el capítulo 4, la llamada "paradoja de la hipótesis añadida" (véanse las pp. 81-84 y 95-97 y sigs.).

Ruptura y continuidad de la tradición, y de sus métodos, se dan cita en estos dos capítulos, sin que dé la impresión de que tan solo estamos ante problemas ocasionales de ciertas teorías dispersas en el pensamiento filosófico sobre la ciencia. Suárez se ocupa de un problema por vez. Las teorías de la explicación y de la confirmación se tratan sucesivamente, pero se exponen como trabajos desarrollados en paralelo de tal modo que la evolución histórica de la corriente lógico-positivista queda manifiesta y se destacan los dos sentidos ya expuestos: su continuidad (la corriente da lugar a un foco de problemas y una fuente de soluciones) y su discontinuidad (las mejoras en los dos ámbitos dependen de una alternativa dialéctica a los fundamentos del positivismo lógico). Poco más se puede pedir desde una perspectiva pedagógica, breve, sistemática y, a la par, respetuosa con la preferencia por la primera tesis, la de naturaleza histórica, arriba mencionada.

La segunda parte mantiene el tono histórico. Se ocupa, en especial, del realismo científico y de las posturas enfrentadas a este y, así, antirrealistas. Observamos de nuevo en ella matices acerca de la propia evolución de la corriente. Un ejemplo de ello es el cambio en actitud hacia las tesis principales del realismo científico que se produce a partir de los años ochenta del siglo pasado, cuando dicha postura pasa de sostener, según Suárez, una tesis filosófica sobre la ciencia presente a otra ligeramente diferente acerca del "objetivo ideal de la ciencia", en palabras del autor: "una afirmación acerca no tanto del estado actual de la ciencia, sino acerca de su objetivo metodológico final" (p. 160); un segundo ejemplo es la evolución en la comprensión del concepto de explicación que requiere la misma corriente y que va más allá de la perspectiva neopositivista, basada en la conocida como "tesis de simetría” (p. 175); un tercer ejemplo son las conexiones biográficas entre los representantes principales del realismo y antirrealismo científicos y sus escuelas y mentores de raíz lógico-positivista (pp. 202-203). Suárez mantiene, de ese modo,

\footnotetext{
8 Suárez describe dicha tesis en las pp. 64-66. Véase también Hempel 1942/1965, p. 234, y Curd y Cover (eds.) 1998, pp. 773-774.
} 
dicho tono histórico, si bien lo combina con un desglose paulatino y sistemático de las tesis que conforman esencialmente la corriente filosófica del realismo científico. A este respecto, el autor dedica cuatro de los cinco capítulos (caps. 7-10) que componen la segunda parte del libro, de título "Epistemología contemporánea de la ciencia" (p. 103 y sigs.), a conseguir ese doble efecto. Y precisamente en esa dimensión sistemática se observa la preferencia por la segunda tesis, de corte filosófico, de la que hablábamos en un principio: el realismo experimental.

El primero de los capítulos de la segunda parte, el sexto, está dedicado a cuatro figuras fundamentales de la filosofía de la ciencia de los comienzos de la segunda mitad del siglo XX: Karl Popper, Thomas Kuhn, Imre Lakatos y Paul Feyerabend. Cada uno de los cuatro merecería un capítulo por separado, pero, de acuerdo con las extensiones dedicadas, antes y después, a otros autores y corrientes, el tratamiento es equilibrado. Por otro lado, Suárez ofrece una versión sumaria de cada uno de ellos completamente útil para una introducción inicial. Dicho tratamiento no está exento de comentarios acerca de las relaciones con el empirismo lógico precedente (a veces paralelo). Así ocurre con Popper y su crítica "interna" — por usar el término de Victor Kraft citado por Suárez (p. 109)— a las tesis del Círculo de Viena; ${ }^{9}$ o las conocidas semejanzas de las obras tardías de Kuhn y de Carnap apuntadas por el autor (pp. 116-117); y ya mencionamos antes una conexión con la obra de Feyerabend destacada por Suárez. Los intentos de conexión son frecuentes y bienvenidos.

La presentación de dichos autores, tanto en este capítulo 6 como en la conclusión del libro (pp. 201-204), es precisa, pedagógica y útil. Es posible, sin embargo, estar en desacuerdo con algunas afirmaciones realizadas por Suárez; mencionaré tres de ellas. La primera, de cariz general, es la idea, comentada en la conclusión, de que el positivismo lógico se caracteriza por una atención a la diversidad de las "prácticas científicas en su contexto histórico" (p. 203) que no hallamos en autores como Kuhn, en quien, al igual que en otros, dicha práctica "queda relegada a una mera comparsa, consecuencia, o ilustración del método" (p. 202). Una lectura de la principal obra de Kuhn, The Structure of Scientific Revolutions, y en especial de su conocida frase de apertura de la sección I, en la que Kuhn dice que "[s]i se considerase como algo más que un acervo de anécdotas o como algo más que mera cronología, la historia podría provocar una transformación decisiva

\footnotetext{
9 Véanse las pp. 109-113 del libro, donde las comparaciones y relaciones críticas con dicha corriente son frecuentes.
} 
en la imagen de la ciencia que ahora nos domina", ${ }^{10}$ pone en duda esa descripción. Si por algo se caracteriza la obra de Kuhn — y podría decirse lo mismo de Lakatos y de Feyerabend - es por emplear la historia de la ciencia como un modo de desmentir una versión artificial del desarrollo científico (la versión de la "ciencia de libro de texto") y de apoyar la descripción del perfil filosófico de la ciencia en la familiarización, precisamente, con la práctica de la ciencia en su contexto real. ${ }^{11}$

La segunda tendría que ver con la adscripción de irracionalidad a los procesos de cambio y elección de paradigma en el libro (véanse diversos pasajes a lo largo de las pp. 114-117, 121 y 128), una crítica ya aducida con frecuencia en contra de los argumentos de Structure. Kuhn menciona a autores como Dudley Shapere o Israel Scheffler como buenos ejemplos de esa clase de crítica. Pero esta afirmación ya fue suficientemente respondida por Kuhn en su momento, incluso en la segunda edición de Structure, en su "Postscript-1969". ${ }^{12}$ Tercero, la afirmación de que Kuhn "no aprecia progreso científico" (p. 119) no es muy consistente con una lectura de la sección XIII de Structure. Una versión más precisa debería especificar que Kuhn corrige la comprensión del progreso científico y defiende, en su lugar, un proceso no teleológico y de corte instrumental y, en cierta medida, evolucionista. ${ }^{13}$

Carnap se mostró de acuerdo, en una conocida carta a Kuhn de abril de 1962, tras leer el manuscrito de Structure, en que el progreso instrumental de la ciencia de corte darwinista que este defendía era muy semejante a su propia propuesta. ${ }^{14}$ Los paralelismos son significativos, como hemos visto que indica también Suárez. Sin embargo, las diferencias de fondo son notables. Kuhn, al igual que Lakatos y Feyerabend, encajan solo con dificultades en los supuestos de evaluación epistemológica asumidos desde el positivismo lógico al realismo

10 Kuhn 1962/1996, p. 1; trad. cast.: Kuhn 2006, p. 57.

11 Véase, e.g., el texto de Joseph Rouse, "Kuhn's Philosophy of Scientific Practice" (Rouse 2003, pp. 107-108 y 112-118), para un estudio a fondo de esta relación esencial del trabajo de Kuhn con las prácticas. En palabras de Rouse, "Kuhn reorienta la filosofía de la ciencia hacia una explicación de las prácticas científicas más que del conocimiento científico" (Rouse 2003, pp. 115-116). Sobre la "ciencia de libro de texto", véase Kuhn 1962/1996, pp. 136-138, y su traducción en Kuhn 2006, pp. 248-250.

12 Véase Kuhn 1962/1996, pp. 185-186, donde también se menciona a Shapere y Scheffler, al igual que las pp. 152-159 de la primera edición; véanse también Kuhn 1977 y 1983/2000 y Mayoral 2012, pp. 265-266.

13 Véase Kuhn 1962/1996, pp. 166-173, Hoyningen-Huene 1993, pp. 258-264, así como la interesante discusión crítica de Bird 2007.

14 R. Carnap a T. S. Kuhn, 28 de abril de 1962, reproducida en Reisch 1991, p. 267; véase también Mayoral 2017, p. 122. 
científico. ${ }^{15}$ Un examen que no aluda a dichas diferencias oculta el trasfondo que permite verlos como teóricos que ofrecen otras perspectivas sobre nociones tan queridas, entonces y ahora, como las de racionalidad o progreso. Estos autores, en resumen, se pueden estudiar como reacciones al positivismo lógico, pero también como fundadores de una rama de la filosofía de la ciencia que, más allá de presentar un frente crítico, contribuye a la pluralidad de dicha disciplina. Esta pluralidad, sin embargo, no está demasiado presente en este libro, que, pese a su enfoque histórico, digno de mención, presenta de todos modos un desarrollo considerablemente lineal.

Tales autores, no obstante, no son el plato fuerte de la segunda parte. Como dijimos, dicha parte se centra sobre todo en exponer el realismo científico, sus críticos y alternativas y una perspectiva en la que el autor se integra, el realismo experimental. Así pues, parte del atractivo de dichos capítulos radica en que encontramos en ellos una caracterización precisa del realismo científico. Algunos de los argumentos y tesis fundamentales para la perspectiva actual nacen de trabajos de Richard Boyd y de Hilary Putnam y debemos incluir a Philip Kitcher y a Stathis Psillos como representantes de la postura actual. ${ }^{16}$ El objetivo de esta corriente es, como bien muestra Suárez, superar la falta de expectativa lógicopositivista de una interpretación literal de los términos teóricos y asumir que estos refieren entidades existentes en un mundo independiente de la mente y, ante todo, que las teorías que emplean dichos términos en sus enunciados son parcial o aproximadamente verdaderas. Nuestras teorías científicas, en otras palabras, nos proporcionan el mejor ejemplo del conocimiento de un mundo que, pese a ser independiente de la mente, nos resulta inteligible gracias a la versión que aquellas nos aportan de este, lo que incluye un vocabulario que, aunque verse sobre lo inobservable, deberíamos interpretar de forma literal. ${ }^{17}$ "Literalidad" (p. 151), "independencia" (del mundo con respecto a la mente; p. 155) y "accesibilidad" (p. 156) son los términos que Suárez emplea para exponer esta visión realista en torno a los tres principios fundamentales correspondientes. ${ }^{18}$

\footnotetext{
15 Sobre el caso de Kuhn, véase Mayoral 2015.

16 Véanse los caps. 7-10 del libro de Suárez, esp. pp. 132 y 140.

17 Como ya dijimos, esta postura puede ser acerca de las teorías presentes o solo una expectativa de la clase de teoría que desearíamos obtener en un límite ideal. Esta otra caracterización se aproxima mucho a lo que Paul Hoyningen-Huene denomina "realismo peirceano"; véase Hoyningen-Huene 1993, p. 56.

18 Véanse los caps. 7-10 del libro de Suárez, esp. pp. 132 y 140.
} 
Tal como Suárez expone, una buena defensa del realismo científico se basa en el llamado "argumento de los milagros" de Putnam, que dice que "el realismo es la única filosofía que no hace del éxito de la ciencia un milagro" (Putnam 1975, p. 73). Suárez explica este argumento al principio del capítulo 7 (pp. 132-135). Dado que la tesis alude a que la utilidad explicativa del realismo científico actúa en defensa de su verdad, se puede formular en términos de un argumento de naturaleza abductiva. Para explicarlo brevemente, partamos del concepto de éxito de la ciencia, algo históricamente constatable. Este es un concepto que incluye toda la evidencia a favor de naturaleza observacional con la que cuentan las teorías científicas y, en especial, las predicciones que siguen de ella, al igual que el ámbito de su aplicación técnica. El argumento abductivo se basa en que la tesis (realista) de que las teorías científicas son (cuando menos, aproximadamente) verdaderas no tiene serios competidores a la hora de explicar convenientemente dicho éxito. ${ }^{19}$

Como muestra Suárez, el realismo científico tiene sus opositores. El autor del libro dedica espacio (pp. 162-167 y 174-177) a mostrar perspectivas contrarias al realismo científico y, entre ellas, se encuentra la de B. C. Van Fraassen, quien, como dice Suárez (p. 178), disocia el concepto de adecuación empírica del de verdad teórica (aun aproximada) y deja claro que solo estamos en disposición de afirmar la primera, pero no la segunda como consecuencia de ello. De este modo, Van Fraassen es antirrealista y, como dice Suárez, "es agnóstico [...] acerca de las entidades no-observables postuladas en la ciencia" (p. 164; véanse también las pp. 162-163). Arthur Fine, por otro lado —al tiempo que el citado Van Fraassen (pp. 163-164)—, es crítico con la inferencia abductiva. Una crítica recurrente es que la inferencia abductiva no es lógicamente válida y que la defensa del realismo científico en términos de esta depende de la idea, no aceptada por el antirrealista, de que una teoría es verdadera porque tiene capacidad explicativa. Sin embargo, esta idea es dudosa y depende de la propia inferencia abductiva, que basa la atribución de verdad a una hipótesis en sus virtudes explicativas. Para antirrealistas como Van Fraassen, esas virtudes no son epistémicas, sino pragmáticas (pp. 175 y 194); y, pese a lo asumido por los realistas, muchos casos en ciencia implican una opción por teorías que, aun siendo empíricamente adecuadas y con ciertas

19 Véanse las pp. 139, 169-174 y 189-190 del libro de Suárez para la presentación de este argumento en su forma abductiva y las pp. 169-170 para las raíces de la inferencia abductiva en el pensamiento de Charles S. Peirce. Para la caracterización aquí ofrecida del éxito de la ciencia, véase Nola y Sankey 2007, pp. 342-343. Sobre el realismo científico y el argumento de los milagros, véase también Chakravartty 2017, sec. 2.1. 
capacidades de explicación, se saben falsas. Como indica Suárez, si la explicación en las teorías científicas no es garantía de su verdad, ¿por qué habría de funcionar de ese modo en el caso de la defensa del propio realismo científico? En ausencia de un apoyo independiente de la abducción, el realista científico se queda, a juicio de antirrealistas como los nombrados, sin una defensa plausible. ${ }^{20}$

Una alternativa a este tipo de realismo científico, que confía en que la verdad teórica (incluso la meramente aproximada) se sigue de sus virtudes explicativas, es un tipo de realismo que, en lugar de centrarse en las teorías y en las inferencias que nos hablan de su verdad, presta atención a dichas entidades en cuestión. Este es el realismo de entidades, o experimental, que expone Suárez en su capítulo 10. Su inferencia, de este modo, no es de las virtudes explicativas de la teoría a la verdad, sino de la posibilidad de que una estructura causal dé cuenta de ciertos fenómenos como sus efectos a la afirmación de la existencia de dicha estructura causal. Esta es la que se conoce como "inferencia a la causa más probable" y se compromete, como señala Suárez, con que es posible construir inferencias en el modo material, más que en el formal, del lenguaje (una distinción introducida por Rudolf Carnap) y con que la mencionada inferencia causal se construye en ese primer modo. ${ }^{21} \mathrm{El}$ tipo de explicación propuesta, muestra Suárez, es óntica, no epistémica (véanse las pp. 191-193); en otras palabras, un hecho no se explica en virtud de la subsunción de su descripción en una estructura teórica, sino porque es parte de una "estructura causal", como dice el autor, "que, de manera operativa, genera, en efecto, tal fenómeno" (p. 193). Autores como Nancy Cartwright (pp. 194-197), Ian Hacking (pp. 197-200) o el propio Suárez (véase p. 187) son defensores, desde enfoques ligeramente diferentes, de este punto de vista realista, no exento tampoco de críticas, por supuesto (véase, e.g., la p. 197).

Mediante este repaso a los contenidos de su último capítulo acabamos con nuestro recorrido por este interesante texto. Podemos concluir subrayando que el libro de Suárez es una magnífica contribución a la bibliografía sobre filosofía de la ciencia en nuestro país. Es un texto con voluntad de enseñanza a nuevas generaciones de filósofos y a cualquier interesado en la materia, sea cual sea su formación; es, de hecho, una buena introducción a esta materia para aquellos científicos que intenten averiguar de manera concisa pero no superficial en qué consisten algunos de los problemas fundamentales de la disciplina y de sus métodos más empleados. Pero también es un texto que ofrece una versión completa, en

20 Para este argumento antirrealista, véanse esp. las pp. 173-175.

${ }^{21}$ Véanse las pp. 187-191 y, sobre la mencionada inferencia, pp. 194 y sigs. 
un nivel principalmente formativo, de todo un modo de hacer filosofía de la ciencia que recorrió todo el siglo XX y que continúa hoy día. Hemos destacado algunos desacuerdos, principalmente en torno la presentación en el libro de las perspectivas de algunos críticos de ese enfoque (o enfoques hermanados) de la filosofía de la ciencia y acerca del carácter algo lineal de la historia descrita en este manual. Pero nada de ello importa demasiado en comparación con lo que Suárez ha logrado aportar: una introducción concisa y profunda, con sensibilidad histórica, al lenguaje en el que se habla y practica la filosofía de la ciencia de hoy en día. El libro tiene otras virtudes, pero ya solo por eso es digno de adquirir y leer con detenimiento.

\section{Juan Vicente Mayoral de Lucas \\ Universidad de Zaragoza \\ jmayoral@unizar.es}

\section{BIBLIOGRAFÍA}

BirD, A. (2007): "What Is Scientific Progress?”, Noûs, 41, pp. 92-117.

Chakravartty, A. (2017): "Scientific Realism", en Stanford Encyclopedia of Philosophy, E. N. Zalta (ed.). URL: <https://plato.stanford.edu/archives/sum2017/entries/ scientific-realism/> (Acceso: 30 de noviembre de 2019)

Creath, R. (2017): "Logical Empiricism", en Stanford Encyclopedia of Pbilosophy, E. N. Zalta (ed.). URL: <https://plato.stanford.edu/archives/fall2017/entries/logicalempiricism/> (Acceso: 30 de noviembre de 2019).

Curd, M., y Cover, J. A. (eds.) (1998): Philosophy of Science: The Central Issues, Nueva York: W. W. Norton.

Earman, J. (1993): "Carnap, Kuhn and the Philosophy of Scientific Methodology", en P. Horwich (ed.), World Changes: Thomas Kubn and the Nature of Science, Cambridge, Mass.: The MIT Press, pp. 9-36.

Friedman, M. (1999): Reconsidering Logical Positivism, Cambridge: Cambridge University Press. Hempel, C. G. (1942/1965): “The Function of General Laws in History”, en Aspects of Scientific Explanation and other Essays in the Philosophy of Science, Nueva York: Free Press, pp. 231-243.

Hempel, C. G (1945/1965): "Studies in the Logic of Confirmation", en Aspects of Scientific Explanation and other Essays in the Pbilosophy of Science, Nueva York: Free Press, pp. 3-46.

Hoyningen-Huene, P. (1993): Reconstructing Scientific Revolutions, Prólogo de T. S. Kuhn, trad. A. T. Levine, Chicago: The University of Chicago Press.

Kunn, T. S. (1962/1996): The Structure of Scientific Revolutions, $3^{a}$ ed., Chicago: The University of Chicago Press. 
Kunn, T. S. (1977): “Objectivity, Value Judgment and Theory Choice”, en The Essential Tension, Chicago: The University of Chicago Press, pp. 320-339.

Kunn, T. S. (1983/2000): "Rationality and Theory Choice", en The Road since Structure, J. Conant y J. Haugeland (eds.), Chicago: The University of Chicago Press, pp. 208-215.

KuHn, T. S. (2006): La estructura de las revoluciones cientificas, $3^{\text {a }}$ ed. cast., trad. e introducción de Carlos Solís, México: FCE.

Mayoral, J. V. (2012): "Five Decades of Structure: A Retrospective View", Theoria, 75, pp. 261-280.

Mayoral, J. V. (2015): "Significado, conocimiento y creencia en Kuhn: la influencia de Wittgenstein y Austin”, en D. Pérez Chico y J. V. Mayoral (eds.), Wittgenstein: la superación del escepticismo, Madrid: Plaza y Valdés, pp. 177-227.

Mayoral, J. V. (2017): “Mundos fenoménicos y léxicos científicos: el relativismo lingüístico de Thomas Kuhn", Revista de Filosofía, 42, pp. 117-134.

Nola, R., y SANkey, H. (2007): Theories of Scientific Method: An Introduction, Stocksfield: Acumen.

Peschard, I. F., y Van Fraassen, B. C. (2018): "Introduction", en I. F. Peschard y B. C. Van Fraassen (eds.), The Experimental Side of Modeling, Minnesota Studies in the Philosophy of Science, vol. 21, Minneapolis: University of Minnesota Press, pp. 1-58.

Putnam, H. (1975): "What Is Mathematical Truth?”, en Mathematics, Matter and Method: Philosophical Papers, Vol. I, Cambridge: Cambridge University Press, pp. 60-78.

Reisch, G. A. (1991): “Did Kuhn Kill Logical Empiricism?” Philosophy of Science, 58, pp. 264-277.

Rouse, J. (2003): “Kuhn's Philosophy of Scientific Practice”, en T. Nickles (ed.), Thomas Kubn, Cambridge: Cambridge University Press, pp. 101-121. 